\title{
Transatlantica
}

Revue d'études américaines. American Studies Journal

$2 \mid 2011$

Sport et société / Animals and the American

Imagination

\section{Le faune et la sirène : la situation de Cuvier dans \\ l'économie de The Marble Faun, de Nathaniel \\ Hawthorne}

Antoine Traisnel

(2) OpenEdition

Journals

Édition électronique

URL : https://journals.openedition.org/transatlantica/5563

DOI : 10.4000/transatlantica.5563

ISSN : 1765-2766

Éditeur

Association française d'Etudes Américaines (AFEA)

Référence électronique

Antoine Traisnel, « Le faune et la sirène : la situation de Cuvier dans l'économie de The Marble Faun, de Nathaniel Hawthorne », Transatlantica [En ligne], 2 | 2011, mis en ligne le 05 juin 2012, consulté le 04 février 2023. URL : http://journals.openedition.org/transatlantica/5563 ; DOI : https://doi.org/10.4000/ transatlantica. 5563

Ce document a été généré automatiquement le 4 février 2023

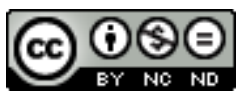

Creative Commons - Attribution - Pas d'Utilisation Commerciale - Pas de Modification 4.0 International - CC BY-NC-ND 4.0

https://creativecommons.org/licenses/by-nc-nd/4.0/ 


\title{
Le faune et la sirène : la situation de Cuvier dans l'économie de The Marble Faun, de Nathaniel Hawthorne
}

\author{
Antoine Traisnel
}

He gave Miriam the idea of a being not precisely man, nor yet a child, but, in a high and beautiful sense, an animal; a creature in a state of development less than what mankind has attained, yet the more perfect within itself for that very deficiency.

Nathaniel Hawthorne, The Marble Faun, $78^{1}$

Le point de départ de cet article est un nom propre dont l'évocation, à la toute fin de The Marble Faun, menace de mettre en péril la fiction. Ce nom, «Cuvier », est celui d'un homme de science dont les travaux sur la classification des espèces animales ont radicalement transformé la manière dont l'Occident conçoit ce qu'on a coutume d'appeler l'« histoire naturelle». Tel que l'emploie Nathaniel Hawthorne, ce nom ne désigne pas tant la figure empirique du scientifique français qu'il ne résume et invoque l'épistémè moderne qui, d'après le Foucault de Les mots et les choses, se fait jour au tournant du dix-neuvième siècle. Dans l'économie de The Marble Faun, "Cuvier » devient la signature d'une vision taxonomique du monde moins préoccupée par les affinités reliant les êtres qui le peuplent que par ce qui les sépare et les constitue en propre. Le nom du père de l'anatomie comparée s'offre dès lors comme l'indice de la résistance qu'oppose la romance à une certaine logique du propre. En effet, si l'on en croit l'« Auteur », ce patronyme et l'idéologie qu'il véhicule signent la faillite de l'esthétique de la réticence et du non-dévoilement mise en œuvre par The Marble Faun.

The idea of the modern Faun loses all the poetry and beauty which the Author fancied in it, and becomes nothing better than a grotesque absurdity, if we bring it into the actual light of day. He had hoped to mystify this anomalous creature 
between the Real and the Fantastic, in such a manner that the reader's sympathies might be excited to a certain pleasurable degree, without impelling him to ask how Cuvier would have classified poor Donatello, or to insist upon being told, in so many words, whether he had furry ears or no. As respects all who ask such questions, the book is, to that extent, a failure. (463-64, je souligne)

L'attention portée aux zones liminales ou anomales qui rendent poreuse la frontière entre réel et fantastique sont des thèmes chers au narrateur de "The Custom House " et amplement discutés par la critique hawthornienne. Ce qui m'intéresse davantage dans ce passage, c'est la référence au Baron Jean Léopold Chrétien Frédéric Dagobert Cuvier, aka Georges Cuvier, dans un livre qui paraît en 1860, quelques mois seulement après On the Origin of Species by Means of Natural Selection, or the Preservation of Favoured Races in the Struggle for Life de Charles Darwin.

Il n'est bien entendu pas question de suggérer que Hawthorne se livre à une critique des travaux du naturaliste français à l'aune d'un livre qu'il n'a pas pu avoir lu au moment où paraît sa romance ${ }^{2}$, mais plutôt de se demander : de quoi Cuvier est-il le nom? À ma connaissance, Hawthorne n'a pas consulté les Leçons d'anatomie comparée. En revanche, il est certain que le nom du naturaliste circulait à l'époque dans les milieux littéraires américains puisqu'on en retrouve la trace chez Emerson ("The American Scholar»), Thoreau (Journal) ou Melville (Moby-Dick), mais aussi dans «The Murders in the Rue Morgue » (1841) de Poe, où l'orang-outang qui tue la fille et la mère L'Espanaye est identifié comme « la bête de Cuvier ». Cette nouvelle fait singulièrement écho à The Marble Faun en ceci qu'il manque à la police pour résoudre le double meurtre (mais s'agit-il d'un meurtre ?) la faculté d'« imagination » dont est doté le Chevalier Dupin pour ouvrir l'investigation à la possibilité du non-humain. L'animal est là où on ne l'attend pas. Il ne peut commettre de meurtre car il hante les marges d'un univers anthropocentré qu'il n'habite plus. Ce caractère spectral confère à l'animal la capacité de pénétrer un espace fermé de l'intérieur car nous avons oublié, semble dire la nouvelle, qu'il était toujours déjà là, parmi nous - nous animaux humains - dans la chambre close de nos esprits cartésiens. L'imagination est un élément indispensable à la résolution du meurtre dans le récit de Poe, mais, de manière significative, c'est la description minutieuse que fait Cuvier du grand orang-outang fauve des îles de l'Inde orientale qui rend possible la capture de l'animal (Poe, 304-05).

Dans l'imaginaire collectif, Cuvier est associé aux tableaux à double seuil capables de contenir et hiérarchiser la diversité du monde. Son nom seul évoque un tournant majeur dans la manière qu'a eue l'homme de se situer par rapport aux autres animaux ${ }^{3}$. «[C]réationniste et fixiste» (Fontenay, 619), Cuvier se dressa contre les théories continuistes de Buffon et de Lamarck. En effet, pour le naturaliste, l'espèce est censée reposer sur un donné offert à l'expérience. Le reste n'est qu'hypothèse, construction, fiction (Foucault, 900). C'est sans doute l'apriorisme invétéré du scientifique qui incita Hawthorne à ériger l'entreprise de Cuvier en contre-exemple de l'esthétique de The Marble Faun, une esthétique qu'on pourrait qualifier d'irréaliste en opposition au réalisme d'un Balzac ${ }^{4}$. De même que Darwin exploite les failles du système de classification de Cuvier pour « effacer le seuil épistémologique et montrer que, en fait, ce qu'il faut commencer par connaître, c'est l'individu avec les variations individuelles" et non l'espèce (Foucault, 901), de même que Darwin substitue à l'identification taxinomique la notion dynamique de généalogie, Hawthorne semble jouer avec les attentes rationalistes et réalistes du lecteur en leur opposant 
l'incertitude et l'ignorance comme régimes d'écriture propices à la romance et en faisant de l'inquiétude épistémologique la matrice de son récit.

\section{La sirène de Cuvier (épistémophilie)}

5 Dans un petit texte intitulé « Recherches anatomiques sur les reptiles regardés encore comme douteux par les naturalistes » lu à l'Institut National de Paris en janvier 1807, soit deux ans après la publication des Leçons d'anatomie comparée qui inscriront son nom au panthéon des zoologistes, Cuvier se penche sur le cas de quelques créatures aquatiques récalcitrantes. Grâce à la dissection, dont il raffine l'art et démocratise la pratique, le naturaliste parvient à démontrer qu'un spécimen rangé par Linné parmi les amphibiens permanents n'est en réalité qu'un vulgaire poisson. "Le grand Linné », comme il l'appelle, avait pris ses vessies pour des poumons.

6 À ce jour, explique Cuvier dans l'introduction, on ne connaît d'animaux amphibies que sous forme de larves dont les branchies s'oblitèrent à mesure que se développent les poumons. L'amphibologie de l'animal amphibie ne serait qu'un état transitoire. Cuvier va alors s'intéresser au cas de trois « reptiles douteux» venus du Nouveau Continent qui possèdent simultanément les deux types d'organes respiratoires. Or, étant donné leur grande taille, il n'est pas certain que ces créatures ne soient que les larves de reptiles adultes. Le premier spécimen auquel Cuvier s'intéresse est affublé du doux nom de «sirène » en raison des deux pattes avant et de la longue queue dont il est flanqué. Envoyé depuis les Amériques à Linné en 1765, cet animal au nom mythique est classé par le naturaliste suédois sous le nom d'« Amphibia meantes » en raison de six critères dont Cuvier établira dans son texte que seul le premier - la créature n'a pas de jambes arrières - est scientifiquement recevable. En 1785, le Dr. Camper, un médecin hollandais que Cuvier admire pour ses intuitions mais à qui il reproche son dilettantisme, étudie un spécimen de sirène au Muséum britannique de Londres. N'apercevant pas de poumons, lesquels étaient sans doute atrophiés en raison d'une mauvaise conservation, Camper classe l'individu parmi les poissons.

7 Afin de trancher le différend entre ses deux prédécesseurs, Cuvier décide de se faire livrer une sirène en 1800. L'autopsie établit que l'animal possède bien à la fois des branchies et des poumons, et que ses appendices latéraux sont des pattes et non des nageoires. Reste à savoir s'il s'agit d'un têtard ou d'un amphibie permanent, une question à laquelle seule l'ostéologie peut répondre de façon définitive. Après une description minutieuse de la charpente osseuse de l'animal, Cuvier conclut qu'il ne s'agit pas d'une larve mais d'un batracien adulte: «En dernier résultat, le présent mémoire prouverait donc que parmi les trois reptiles regardés encore récemment comme douteux (Sirène, Protée, Axolotl) un seulement, à savoir l'Axolotl, doit être effacé du catalogue des animaux et considéré comme une larve $»^{5}$. Les deux autres sont dignes de figurer dans le «catalogue " établi par le scientifique. Afin d'ôter tout " doute », le scientifique décide de créer un nouveau genre, les pérennibranches, parmi les batraciens urodèles (qui gardent leur queue à l'état adulte). C'est ainsi qu'opère Cuvier : pour classer l'inclassifiable, il ajoute une colonne à l'inventaire taxinomique de l'histoire naturelle. Et la sirène de quitter les eaux mythiques du détroit de Messine pour intégrer les tableaux classificatoires de la science moderne.

Dans L'ouvert, Giorgio Agamben rappelle que les sirènes côtoyaient encore les phoques et les lions de mer dans le traité du naturaliste suédois Peter Artedi, le père de 
l'ichtyologie ironiquement mort noyé en 1735 à l'âge de trente ans ${ }^{6}$ (Agamben, 45). Après Cuvier, telle juxtaposition n'est plus possible, même si celui-ci considère Artedi comme l'une des figures majeures de l'ichtyologie positive :

Nous reconnaissons dans les progrès de l'ichtyologie trois époques principales. Elle ne se compose d'abord, pendant bien des siècles, comme toutes les branches de l'histoire de la nature, que d'observations partielles. [...] Pendant plus de dix-huit cents ans ceux qui écrivent sur l'histoire naturelle se bornent presque à copier Aristote, ou à le commenter : mais au milieu du seizième siècle, Rondelet, Belon et Salvien, reviennent à la véritable observation; et en rectifiant et en étendant ce qu'Aristote avait dit, ils donnent à l'ichtyologie une base positive par des descriptions et des figures d'un certain nombre d'espèces bien déterminées ; à la fin du dix-septième siècle, Willughby et son ami Jean Ray essaient les premiers de distribuer ces espèces d'après une méthode fondée sur des caractères distinctifs tirés de leur organisation; enfin, au milieu du dix-huitième siècle, Artedi et Linnaeus complètent cette entreprise, en établissant des genres bien définis, en y plaçant des espèces certaines et caractérisées avec netteté. Depuis lors, lichtyologie marche sans obstacle vers la perfection... (Cuvier, 1828, 3-4, je souligne)

Bien qu'il se dise héritier de Linné et Artedi, l'exemple de la sirène montre à quel point Cuvier est exemplaire de la rupture épistémologique survenue au début du XIX ${ }^{\mathrm{e}}$ siècle. La passion classificatrice de Cuvier, de laquelle découle une machinerie théorique produisant des distinctions toujours plus précises et des discontinuités toujours plus nombreuses, vient rompre avec la perspective globalisante des pionniers de l'histoire naturelle. Foucault écrit :

Lorsque vous lisez l'Histoire naturelle de Buffon, vous savez très bien de quoi Buffon parle. Pourtant la manière qu'il a de traiter les choses et de poser les problèmes va être complètement bouleversée à partir, en gros, de Cuvier, c'est-à-dire quarante ans après, lorsque, avec l'Anatomie comparée, Cuvier va pouvoir faire un déchiffrement des structures, pouvoir faire des rapprochements, des classifications, des organisations d'un tout autre type. (Foucault, 898)

La méthode classificatoire de Cuvier sera remise en question quelque cinquante ans plus tard par les travaux de Darwin, lequel renoue, sans pour autant tout à fait en reprendre les thèses, avec la perspective transformiste de Lamarck. Dans un article intitulé «La situation de Cuvier dans l'histoire de la biologie », Foucault avance que pour Cuvier, tout était organisé pour et à partir de l'espèce, contrairement à Darwin pour qui l'espèce "n'est pas une réalité originairement première et analytiquement ultime» (Foucault, 898). Darwin voit beaucoup de cas limites où il est difficile de distinguer l'espèce de la variété.

En somme, Darwin admet que tous les cadres taxinomiques que l'on a proposés pour classer les animaux sont, jusqu'à un certain point, des catégories abstraites. Il y a donc, pour lui, une réalité qui est l'individu, une seconde réalité qui est la "variativité" de l'individu, sa capacité à varier. Tout le reste (que ce soit l'espèce, ou le genre, ou l'ordre, etc.) est une sorte de construction qu'on bâtit à partir de cette réalité qu'est l'individu. Dans cette mesure, on peut dire que Darwin s'oppose absolument à Cuvier. (Foucault, 898)

11 «Dans cette mesure» seulement, car Foucault va montrer qu'il est impossible de passer, dans l'histoire de la biologie, directement de Lamarck à Darwin sans prendre en compte l'intervention de Cuvier car la critique de l'espèce chez Lamarck n'est nullement superposable à celle de Darwin. Ce dernier, affirme Foucault, n'a pu élaborer ses théories qu'à partir de la réorganisation du savoir biologique que propose Cuvier. 
Tout en tenant compte de cette nuance importante, je voudrais m'attarder quelques instants sur cette opposition absolue à Cuvier qui rapproche Darwin et Hawthorne.

\section{Contextes de The Marble Faun}

Comme je l'ai mentionné plus haut, L'origine des espèces et The Marble Faun furent publiés à une année d'écart. Dans The Marble Faun, la dernière romance achevée de Hawthorne, l'auteur fait le pari d'introduire une figure mythique - un faune, être hybride mihomme mi-animal - dans le décor minutieusement reconstitué de la Rome contemporaine. L'irruption de cet individu «douteux» soulève la question du scepticisme, qu'Emily Budick érige en principe fondamental de la romance, et interroge les conséquences que l'incertitude épistémologique dans laquelle sont plongés les personnages peut avoir sur leur appréhension d'un environnement partiellement inconnaissable ${ }^{7}$. Cette quasi-coïncidence entre les livres de Darwin et de Hawthorne, dans la date de publication et dans la thématique qu'ils abordent, nous invite naturellement, si j'ose dire, à interroger le rôle que joue l'animalité dans l'économie narrative et dans l'esthétique de The Marble Faun. À bien y regarder, il y a assez peu d'animal chez Hawthorne. À l'exception remarquable du papillon (mécanique) de «The Artist of the Beautiful » ou du singe faisant la grimace au pied de la boîte à musique de The House of the Seven Gables - deux figures animales qui laissent supposer, dans le droit fil de la pensée cartésienne, que l'animal est assimilable à un automate - et de quelques autres $^{8}$, l'animal se fait plutôt rare chez un auteur qui semble privilégier, contrairement à un Cooper, un Emerson ou un Thoreau, les labyrinthes de l'âme humaine au rapport qu'entretient l'homme avec cette forme familière d'altérité qu'est la nature.

13 À première vue, The Marble Faun ne déroge pas à cette règle. L'hybridité du faune ne semble en faire guère plus qu'un représentant marginal de la faune, puisqu'en tant que divinité rustique il renvoie à une animalité idéalisée, de surcroît dans la romance figée dans la pierre. Ceci a sans doute contribué à ce que la figure animale soit étudiée par les critiques de The Marble Faun non pas en tant qu'animal per se mais davantage pour le potentiel métaphorique et heuristique qu'elle recèle. En ceci qu'elle représenterait un degré moindre de l'évolution ("a state of development less than what mankind has attained»78), elle servirait la réflexion morale, politique ou esthétique suscitée par la romance. Par amour pour son amie Miriam, Donatello commet un meurtre qui le fera passer de l'innocence animale à l'expérience humaine : «instead of the wild boy, the thing of sportive, animal nature, the sylvan Faun, here was now the man of feeling and intelligence " (320). La "transformation» du faune ${ }^{9}$, son passage de l'animalité à l'humanité, a été lue comme une réflexion éthique calquée sur le modèle de la felix culpa (Pétillon), suivant une hypothèse que l'ouvrage avance explicitement; on a perçu le faune comme la représentation allégorique des esclaves noirs d'Amérique évoquant la manière dont Hawthorne anticipait les vives tensions qui deux ans plus tard allaient mener les États-Unis à la Guerre de Sécession (Riss); on en a fait le corrélat fictif de l'instabilité générique de la romance (Budick); la chute du faune en est venue à figurer le passage de l'art classique à l'art gothique (Bradbury) ou à l'art moderne (Manning).

Loin de vouloir remettre en cause la validité de ces interprétations, j'aimerais inverser cette tendance critique afin de demander ce que l'esthétique de la romance, telle que l'a esquissée Hawthorne dans les préfaces de ses romances américaines et telle qu'elle est 
mise en pratique dans The Marble Faun, peut nous apprendre sur le rapport entre l'homme et ce qu'on appelle l'« animal ». En d'autres termes, The Marble Faun peut-il offrir les bases d'une réflexion sur la façon dont la faille d'ordre épistémologique qui s'ouvre à l'orée du XIX siècle a marqué l'imaginaire occidental dans son rapport à l'animal, et sur la manière dont la romance, marquée du sceau de l'incertitude et de l'ignorance - voire, peut-être, de la bêtise -, œuvre à réévaluer la complexité négligée de l'énigme animale ? Comment, en somme, le livre pose-t-il « la question de l'animal » au moment où Darwin s'apprête à infliger à l'homme la seconde de ses trois grandes blessures narcissiques ${ }^{10}$ en le privant de "propre » et en conjuguant l'« origine des espèces » au singulier?

J'avance l'idée que The Marble Faun peut se lire comme le théâtre d'un conflit entre, d'un côté, une certaine logique du propre et de la connaissance positive et, de l'autre, une esthétique de l'impureté, de la ruine et de l'inachèvement. Hawthorne disparaît de la scène littéraire en 1852 pour refaire surface en 1860 avec sa romance italienne. D'après la majorité des critiques, l'apogée de la carrière littéraire de Hawthorne, qui correspondrait à la publication de sa trilogie américaine (1850-1852), coïncide parfaitement avec ce que F. O. Matthiessen a nommé la "Renaissance américaine " pour décrire la période comprise entre 1850 et 1855 particulièrement fertile en " chefsd'œuvres ». Un tel découpage de l'histoire culturelle a été dénoncé comme arbitraire mais demeure toutefois la référence pour bon nombre de critiques, qui appréhendent The Marble Faun comme un ouvrage raté attestant le déclin des capacités artistiques de l'écrivain. Ces commentateurs s'inscrivent dans le sillage tracé par Henry James, lequel décrit la romance italienne comme un ouvrage composé de bons passages mais dont l'ensemble s'avère insatisfaisant (James, 165) ${ }^{11}$. Pour Pierre-Yves Pétillon, The Marble Faun n'est "guère, sur fond de décor italien, qu'un remake de "L'Arbre de mai": un roman-musée, dont le personnage central n'est plus qu'un "copiste", et surtout un roman "flou", au sens photographique du terme, qui, bien que publié, garde un air d'inachevé » (Pétillon, 65).

\section{Point de vue (épistémophobie)}

De l'aveu de l'« Auteur » lui-même, l'ouvrage est un échec relatif, mais le reproche que lui fait Pétillon doit être nuancé si l'on tient compte de la possibilité que l'inachèvement de The Marble Faun ne soit pas tant le symptôme de la fatigue ${ }^{12}$ d'un écrivain vieillissant que la marque d'un parti pris esthétique. L'effet de flou que génère la lecture de The Marble Faun résulte, à mon sens, d'un travail minutieux sur le point de vue, qui m'apparaît délibérément approximatif. À la demande des lecteurs britanniques insatisfaits par les nombreuses zones d'ombre du récit, Hawthorne avait accepté d'ajouter une postface explicative à sa romance. Feignant d'accéder à leur requête, l'« Auteur » s'amuse ouvertement de la frustration de ses lecteurs car l'ajout n'éclaire en réalité pas grand-chose. Cette ultime épanorthose ne tient nullement la promesse de sens et de résolution que constitue traditionnellement la postface. L'ironie patente de ce raccroc de dernière minute invite à percevoir l'aspect tronqué du livre comme résultant d'un choix délibéré de la part de l'« Auteur ». Par ailleurs, il est significatif que le livre se termine sur le refus poli de donner au lecteur satisfaction sur une question d'ordre anatomique. 
17 Il s'agit pour le narrateur de savoir si Donatello, un jeune italien simple d'esprit, est la réincarnation du Faune sculpté par Praxitèle. Tout au long du livre, Donatello est comparé à un animal ( I I continually find myself treating Donatello as if he were the merest unfledged chicken " [15]; "there is a great deal of animal nature in him " [135]; etc.). Dans le premier chapitre, les trois artistes qui l'accompagnent, Hilda, Kenyon et Miriam, s'amusent de la ressemblance entre Donatello et la statue. Les seuls indices qui trahissent l'animalité de la statue de marbre sont ses oreilles pointues: "The pointed and furry ears [...] are the sole indications of his wild, forest nature " (10). Désirant vérifier que leur ami partage bien toutes les caractéristiques du faune, ils lui demandent de secouer ses bouclettes pour déterminer dans quelle catégorie le ranger. Réfractaire, Donatello se dérobe à ce strip-tease capillaire et, anticipant la prière que l'« Auteur » adresse au lecteur dans la postface, implore ses amis de ne pas chercher à en savoir trop : "I entreat you to take the tips of my ears for granted ». La créature refuse de se laisser examiner de trop près: " "I shall be like a wolf of the Apennines," he continued [...], "if you touch my ears ever so softly. None of my race could endure it. It has always been a tender point with my forefathers and me » (12).

18 Le jeu sur le "point sensible " que sont les oreilles de la créature laisse affleurer le sous-texte érotique du livre, une dimension que l'écho transparent à l'attirance contrenature qu'éprouve Titania pour les oreilles velues de Bottom dans A Midsummer Night's Dream (IV.1) rend manifeste. Sans trop s'appesantir sur l'aspect phallique des oreilles pointues, rappelons que le terme "point » vient du latin punctum, la piqûre et de pungere, percer, piquer. Miriam, la jeune femme dont Donatello est épris, réplique: " "your tender point-your two tender points, if you have them-shall be safe, so far as I am concerned» (12-13, je souligne). On peut lire cette saillie, tantôt comme le refus de jouer le jeu de la séduction auquel la convie le jeune italien tantôt, suivant une lecture plus généreuse, comme l'acceptation de l'ambiguïté générique de Donatello. Miriam poursuit en anglais, aliénant ainsi davantage le pauvre soupirant :

"But how strange this likeness is, after all!-and how delightful, if it really includes the pointed ears! Oh, it is impossible of course," she continued, in English, "with a real and common-place young man like Donatello; but you see how this peculiarity defines the position of the Faun, and, while putting him where he cannot exactly assert his brotherhood, still disposes us kindly towards the kindred creature. He is not supernatural, but just on the verge of Nature, and yet within it. What is the nameless charm of this idea, Hilda? You can feel it more delicately than I." (13)

19 Le point sur lequel achoppe l'entendement des trois amis est un détail, mais c'est précisément le détail - et l'action de détailler - qui motive et autorise la connaissance taxinomique. À la logique classificatoire, Miriam substitue une autre approche, moins intellectuelle que sensuelle. Le " point » dont il est question s'entend alors comme le punctum barthésien, soit comme un «objet partiel» qui fait vaciller la stabilité épistémologique du studium (La Chambre claire). Le corps de Donatello est foncièrement impur, impropre, innommable (nameless charm), mais il demeure malgré tout approchable. The Marble Faun propose de concevoir le rapport à l'autre non en termes de disparités strictes mais, plutôt, d'affinités. Suggérées dans ce passage par le polyptote kindly/kindred, ces affinités ne sont pas tant de l'ordre de l'intelligible que du sensible (You can feel it more delicately than I). Le point de détail discuté dans ce passage figure l'extrémité à partir de laquelle le corps d'un individu est mesurable et, partant, saisissable dans sa totalité, mais également la limite d'une telle entreprise systématique. Le point est un lieu sans étendue, un espace infime, par définition immesurable. Le 
point détermine la limite d'un corps tandis que lui-même échappe à la logique de la détermination. Le jeu sur la pointe des oreilles du faune n'est pas si innocent qu'il y paraît à première vue. Le faune est doté d'un corps marginal : Not supernatural, but just on the verge of Nature, and yet within it. À la fin du dialogue, il n'est plus tant question de savoir dans quelle catégorie ranger l'être hybride que d'exprimer un ressenti, un point de vue. Point, ici, peut s'entendre comme un adverbe de négation. Point de vue : l'objet échappe à la capture taxonomique et se dérobe à logique du propre.

De même que, dans la diégèse, l'identité de Donatello ne sera jamais révélée à ses amis, dans la postface, le narrateur se prétend incapable de satisfaire les attentes des lecteurs frustrés. Doté d'une omniscience capricieuse, il se voit contraint d'interroger Kenyon, lequel lui fait une réponse pour le moins sibylline :

“Only one question more. Did Donatello's ears resemble those of the Faun of Praxiteles?"

"I know, but may not tell," replied Kenyon, smiling mysteriously. "On that point, at all events, there shall be not one word of explanation." (467)

"Sur ce point »- ou plutôt, au sujet de la pointe des oreilles de Donatello -, il lui faut se taire. L'usage des modaux may et shall indique qu'il s'agit d'un problème moral et non épistémologique. Ainsi, à la manière d'un Bartleby malicieux, Kenyon décourage ce que Barthes nomme «la tentation du sens" et prive le lecteur de tout espoir de connaissance certaine. Le narrateur et le lecteur doivent se contenter d'un sourire énigmatique mêlant connivence et secret. En conférant le savoir à l'un de ses personnages, l'« Auteur » condamne l'entreprise herméneutique à la spéculation et invite le lecteur, à travers le sourire de Kenyon, à prendre une distance amusée avec le texte.

L'ambivalence épistémologique qui hante The Marble Faun trouve son illustration la plus patente dans la scène où les trois artistes s'extasient en chœur sur la ressemblance étonnante entre le Faune de Praxitèle et le jeune italien. Miriam s'exclame: "Our friend Donatello is the very Faun of Praxiteles » (14). Par le truchement de la copule be, elle établit l'identité du modèle et de la copie comme indubitable. Un instant plus tard, la même Miriam confie à Hilda qu'elle craint en réalité d'avoir été influencée par l'enthousiasme de ses amis : «I doubt the reality of this likeness of Donatello to the Faun [...]. To say the truth, it never struck me so forcibly as it did Kenyon and yourself, though I gave in to whatever you were pleased to fancy... » (18) L'identité est alors réduite à une simple ressemblance (likeness), une idée soutenue par l'emploi du terme fancy qui renvoie à la notion romantique d'une imagination défectueuse ${ }^{13}$. Comme en réponse à Miriam, Hilda se lamente quelque cent pages plus loin : « "It annoys me very much, [...] this inclination which most people have, to explain away the wonder and mystery out of everything. Why could not you allow me-and yourself-the satisfaction of thinking him a Faun?" » (104) Le texte hésite entre désir de croyance et tentation de connaissance. Cette oscillation est présente dès la scène initiale, où tandis qu'on lui demande si elle aussi perçoit la ressemblance entre Donatello et le Faune de Praxitèle, Hilda répond: "Not quite-almost-yes, I really think so» (7). Ainsi cousue d'épanorthoses, la romance est ponctuée en sous-main par des retouches qui trahissent l'instabilité épistémologique du monde dans lequel évoluent les personnages. Comme je l'ai suggéré plus haut, si l'animal " encore douteux » qui occupe le centre de l'intrigue éclaire le scepticisme dont est empreinte l'écriture de The Marble Faun, il me semble qu'en retour la romance, non sans précautions, invite le lecteur à se défaire d'une idée 
préconçue par rapport à ce qui constitue son environnement, et plus particulièrement sa relation à l'animal.

\section{Point de fuite}

Alors que Cuvier débarrasse le monde de ses animaux fabuleux et transforme la sirène en crapaud, Hawthorne le réenchante en y introduisant une espèce disparue. Navigant entre anthropomorphisme et thériomorphisme, jouant avec la séduisante monstruosité de l'animal douteux qu'est Donatello, The Marble Faun désarçonne les certitudes de son lectorat en faisant de l'épistémologie cuviérienne un mode inefficace de capture de l'énigme animale - entendue comme " existence rebelle à tout concept » (Derrida, 26), comme ce sur quoi devrait achopper la raison - contrairement au Chevalier Dupin qui a recours à Cuvier pour triompher du grand singe. Il s'agit peut-être d'opposer, suivant Derrida, le savoir philosophique à la pensée poétique (Derrida, 66) et, ce faisant, de favoriser une pensée de la ressemblance et de l'impropre afin de construire avec l'animal des affinités effectives, comme appelle à le faire la biologiste et philosophe Donna Haraway (Haraway, 2003, 43).

Dans "A Cyborg Manifesto ", Haraway explique que les monstres et les créatures hybrides ont toujours servi à définir les frontières de la communauté humaine dans l'imagination occidentale: "The Centaurs and Amazons of ancient Greece established the limits of the centred polls of the Greek male human by their disruption of marriage and boundary, pollutions of the warrior with animality and woman " (Haraway, 1990, 180). Elle réclame un renversement de cette tendance communautariste, arguant que le monstre animal ne constitue pas l'envers négatif de l'humain mais, au contraire, en révèle le potentiel d'hybridation et de création. Dans cette optique, l'animalité, qui dans The Marble Faun est souvent identifiée à une essence protohumaine, ne cesse de hanter ce qui le chasse et constitue à la fois la ruine et le salut de l'humain. Libre au lecteur d'accepter l'invitation à rejoindre, pour un temps, la danse sylvestre (chapitre 10) ou le carnaval ("Hereupon, a whole host of absurd figures surrounded him, pretending to sympathize in [Kenyon's] mishap. Clowns and particoloured harlequins; orang-outangs; bear-headed, bull-headed, and dog-headed individuals... » [446]) et de se perdre un instant dans l'hybride et l'absurde, à l'instar de Miriam qui, contrairement à Hilda et Kenyon, accepte la communauté monstrueuse que lui propose Donatello. Miriam fait l'expérience de l'animalité, soit l'expérience d'une certaine involution. Mais dans The Marble Faun, rien ne dure, pas même les statues de marbre, censées renvoyer à de l'intemporel, qui dans la romance sont en ruines et jaunies par le temps. Les deux amants encourent le risque de passer le point de non-retour en s'engageant vers le non-humain: Donatello finit enfermé - capturé - dans une prison du Vatican, et Miriam fait une dernière apparition muette à la fin de la romance, visiblement accablée par le crime dont elle s'est rendue complice :

[W] hen the kneeling figure beneath the open Eye of the Pantheon arose, she looked towards [Hilda and Kenyon] and extended her hands with a gesture of benediction. Then they knew that it was Miriam. They suffered her to glide out of the portal, however, without a greeting; for those extended hands, even while they blessed, seemed to repel, as if Miriam stood on the other side of a fathomless abyss, and warned them from its verge (461).

L'expérience animale se paye de l'exclusion de la communauté humaine. Hilda et Kenyon quittent l'air impur de l'Italie pour s'en retourner convoler en justes noces aux 
États-Unis, abandonnant derrière eux amis et ambitions artistiques. L'irréalisme que revendique la romance n'est pas tant une libération qu'une condamnation. The Marble Faun est un livredu doute, que le bégaiement incessant de l'écriture redouble et qui ouvre à la possibilité onirique d'une hybridation heureuse («a being in whom both races meet on friendly ground») mais utopique et, par conséquent, condamnée à l'échec. « To-day Donatello was the sylvan Faun; to-day Miriam was his fit companion, a Nymph of grove or fountain; to-morrow-a remorseful man and woman, linked by a marriage bond of crime-they would set forth towards an inevitable goal» (435, je souligne). La structure anapestique de la phrase fait retentir le glas terrible du Jugement Final. Miriam devient muette, contaminée par le mystère silencieux («mute mystery " [10]) qui caractérise l'animal et au nom duquel il est, nous dit Kenyon, impossible de parler, sinon de façon impropre.

Cela signifie-t-il que la romance annonce la victoire de Cuvier? Sans doute, mais s'il est une chose que nous apprend l'ironie de la postface ajoutée après la parution de la romance, c'est qu'aucune fin n'est définitive, et qu'il reste toujours quelque chose, après tout. Car "après tout", nous dit le narrateur, la littérature est peut-être le témoin d'une altérité possible, et le poète celui ou celle qui peut rendre compte d'un temps oublié ou refoulé où le rapport à la nature était plus strict et plus intime: «And, after all, the idea may have been no dream, but rather a poet's reminiscence of a period when man's affinity with Nature was more strict, and his fellowship with every living more intimate and dear» (10-11, je souligne). Temporalité paradoxale du syntagme "après tout", qu'on doit peut-être entendre dans le sens fort d'un épisode apocalyptique. Que reste-t-il, après tout ? La romance pointe vers une temporalité posthistorique, dont L'ouvert fait le temps d'une réconciliation possible entre l'homme et l'animal (Agamben, 13). Relisant la fameuse prédiction de Foucault à la fin de Les Mots et les choses, Agamben propose de concevoir la disparition de l'humain comme un événement heureux, une hybridation à venir qui ne représente pas «une nouvelle déclinaison du rapport homme-animal» (ibid., 147) mais au contraire signale la suspension du découpage inlassable effectué par ce que le philosophe nomme la "machine anthropologique». Cette suspension passe par la formation d'une "zone d'inconnaissance - ou mieux, d'ignoscence » (ibid., 144), d'un au-delà ou d'un en-deçà de la connaissance qui n'est point sans rappeler la réticence pudique de Donatello, de Miriam, de Kenyon et finalement de l'« Auteur » de The Marble Faun.

I know, but may not tell.

On that point, there shall be not one word of explanation.

\section{BIBLIOGRAPHIE}

AGAMBEN, Giorgio, L'ouvert. De l'homme et de l'animal (2002), traduit de l'italien par Joël Gayraud, Paris, Payot \& Rivages, 2006.

BARTHES, Roland, La Chambre claire. Notes sur la photographie, Paris, Gallimard, 1980. 
BAYM, Nina, «The Marble Faun : Hawthorne's Elegy for Art », The New England Quarterly, vol. 44, No. 3, September 1971, 355-76.

BRADBURY, Malcolm, Preface to The Marble Faun, London, Everyman's Library, 1995.

BRODHEAD, Richard, Introduction and Notes to The Marble Faun, New York, Penguin Classics, 1990.

BUDICK, Emily Miller, « Perplexity, Sympathy, and the Question of the Human : A Reading of The Marble Faun ", in The Cambridge Companion to Nathaniel Hawthorne, édité par Richard Millington, Cambridge University Press, 2004, 230-50.

CUVIER, Georges et Achilles VALENCIENNES, Histoire naturelle des poissons, tome premier, Paris, Levrault, 1828.

CUVIER, Georges, Leçons d'anatomie comparée, recueillies et publiées par M. Duméril, seconde édition, corrigée et augmentée, tome premier, Paris, Imprimerie d'Hippolyte Tilliard, 1835.

---, « Recherches anatomiques sur les reptiles regardés encore comme douteux par les naturalistes, faite à l'occasion de l'axolotl rapporté par M. de Humboldt du Mexique », Paris, 1807.

DECHAMBRE, Amédée, Dictionnaire encyclopédique des sciences médicales, volume 88, Paris, Masson, 1881.

DERRIDA, Jacques, L'animal que donc je suis, Paris, Galilée, 2006.

FONTENAY, Elizabeth de, Le Silence des bêtes. La Philosophie à l'épreuve de l'animalité. Paris, Fayard, 1998.

FOUCAULT, Michel, Dits et Écrits, Volume 1, Paris, Gallimard, 2001.

HARAWAY, Donna, Simians, Cyborgs and Women, New York, Routledge, 1990.

---, The Companion Species Manifesto, Chicago, Prickly Paradigm Press, 2003.

HAWTHORNE, Nathaniel, The Marble Faun, or the Romance of Monte Beni, Centenary Editions, volume IV, Ohio States University Press, 1971.

JAMES, Henry, Hawthorne, London, Macmillan and Co, 1879.

MANNING, Susan, Preface to The Marble Faun, Oxford, Oxford University Press, 2002.

PÉTILLON, Pierre-Yves, Présentation et postface des Contes et Récits de Nathaniel Hawthorne, Traduction de Muriel Zagha, Paris, Imprimerie Nationale Éditions, 1996.

POE, Edgar Allan, Great Short Works, introduction de G.R. Thompson, New York, Perennial Library, 1970.

RISS, Arthur, « The Art of Discrimination », ELH, Volume 71, Baltimore, Spring 2004, 251-87.

TAQUET, Philippe, Cuvier. Naissance d'un génie, Paris, Odile Jacob, 2006.

\section{NOTES}

1. Toutes les références à The Marble Faun renvoient à la Centenary Edition, vol. VI.

2. Il serait risqué de faire trop de cas de ce rapprochement, mais on remarque que le vocabulaire utilisé pour décrire la gestation de la romance n'est pas sans évoquer la phylogénèse darwinienne. Hawthorne relate sa rencontre avec la sculpture de Praxitèle dans ces termes : «Its lengthened, but not preposterous ears, and the little tail which we infer, behind, have an exquisite effect, and 
make the spectator smile in his very heart. This race of fauns was the most delightful of all that antiquity imagined. It seems to me that a story, with all sorts of fun and pathos in it, might be contrived on the idea of their species having become intermingled with the human race; a family, with the faun-blo[o]d in them, having prolonged itself from the classic era till our own days. The tail might have disappeared by dint of constant intermarriages with ordinary mortals; but the pretty, hairy ears, should occasionally reappear in members of the family; and the moral instincts and intellectual characteristics of the faun might be most picturesquely brought out, without detriment to the human interest of the story. Fancy this combination in the person of a young lady!» (xxi, je souligne). Hawthorne abandonna le projet de mettre en scène une faunesse, mais il conserva l'idée d'une transmission qui serait non-linéaire et atavique. Il n'est plus question de malédiction, comme c'est le cas avec l'héritage des Pyncheon dans The House of the Seven Gables, mais d'une passation arbitraire d'attributs récessifs ou dominants sur plusieurs générations.

3. Sur cette question, voir Le silence des bêtes d'Elisabeth de Fontenay (Fontenay, 563).

4. Je mentionne Balzac à dessein parce que les édifices logiques mis en place par Cuvier ont servi de modèle à l'élaboration de la Comédie humaine, dans laquelle l'écrivain étudie l'anatomie comparée de la société. «Cuvier n'est-il pas le plus grand poète de notre siècle ? [...] [N]otre immortel naturaliste a reconstruit des mondes avec des os blanchis, a rebâti comme Cadmus des cités avec des dents, a repeuplé mille forêts de tous les mystères de la zoologie avec quelques fragments de houille, a retrouvé des populations de géants dans le pied d'un mammouth. » In La Peau de Chagrin, cité par Philippe Taquet (Taquet, 10).

5. Dans la troisième édition de son Règne animal, Cuvier reviendra à regret sur cette classification et placera l'axolotl parmi les batraciens pérennibranches : "Ce n'est encore qu'avec doute que je place l'Axolotl parmi les genres à branchies persistantes : mais tant de témoins assurent qu'il ne les perd pas que je m'y vois obligé » (cité par Dechambre, 788).

6. Pour son épitaphe, le botaniste George Shaw (1751-1813) écrit: «Here lies poor Artedi, in foreign land pyx'd / Not a man nor a fish, but something betwixt, / Not a man, for his life among fishes he past, / Not a fish, for he perished by water at last. » Cuvier fait référence à Artedi dans son Tableau historique des progrès de l'ichtyologie.

7. "The fiction asks, interrogates, and plays out the central inquiry : how do I know the world exists? How do I know that I exist in it? Unlike other forms of skepticism, however, including some contemporary versions of it within the field of literature and literary criticism, romance fiction asks this question, not in order to dismiss out of hand the possibilities of human knowledge or (even more importantly) of human action in a not-completely knowable world. Rather it pushes this inquiry forward in order to insist on the exquisite difficulty of making just those moral judgments as we human beings, despite the inadequacy of our evidences, are nonetheless required to make but which, because of our doubts, also require that we always take into account our own and other people's subjectivities. » (Budick, 231).

8. Sans faire la liste exhaustive des animaux qui peuplent l'univers hawthornien, on peut mentionner les cochons de The Blithedale Romance, les poules de The House of the Seven Gables, ou encore le loup de "Endicott», sans oublier les créatures symboliques, comme l'aigle de The Scarlet Letter, le serpent de "Egotism or the Bosom Serpent ", et les animaux mythiques de "The Maypole of Merrymount » et des contes pour enfants.

9. La romance a d'abord été publiée en Angleterre sous le titre Transformation.

10. Le mot est de Freud, décrivant les trois grandes découvertes scientifiques qui inquiètent le préjugé anthropocentriste. La première est la révolution copernicienne, qui réfute l'idée que la Terre est au centre de l'univers, et la troisième la psychanalyse, qui avance que l'homme est esclave de ses pulsions.

11. Non sans ironie, dans le chapitre qu'il consacre aux années italiennes, James suggère que l'échec de Hawthorne vient de ce que celui-ci serait le «dernier spécimen» d'une lignée d'écrivains primitifs : «I think of him as the last specimen of the more primitive type of men of letters; 
and when it comes to measuring what he succeeded in being, in his unadulterated form, against what he failed of being, the positive side of the image quite extinguishes the negative. " (James, 162-63, je souligne).

12. C'est la thèse défendue par Nina Baym, qui lit The Marble Faun comme le roman d'apprentissage esthétique de Kenyon (Baym, 356). Cet apprentissage s'apparente à un désapprentissage puisque Kenyon troque le marteau et le burin contre la promesse d'une existence en compagnie de Hilda. Il semblerait à première vue que Kenyon choisisse la vie (conjugale) à la froideur mortifère du marbre. En réalité, explique Frederick Crews, le sculpteur abandonne la voluptueuse Vénus au profit de la pudibonde Hilda. Ce choix marque selon Baym la fin de la carrière artistique de Kenyon. Cette analyse est convaincante, si ce n'est pour sa conclusion qui fait de Kenyon le double fictionnel d'un Hawthorne épuisé et incapable de rivaliser avec son moi idéal. La fragmentation du livre comme celle de la "Venus of the Campagna » à la fin du livre serait pour Baym un geste tristement iconoclaste (366), un simple constat d'échec. Si cette analyse est admise par la grande majorité de la critique, il me semble possible de lire la romance différemment, comme ces pages se proposent de le démontrer.

13. Hawthorne affectionne particulièrement le terme fancy - que l'on retrouve dans le passage où il est fait mention de Cuvier - pour désigner ses fictions. Le sujet de cet article ne se prête pas à une explication détaillée de l'inclination surprenante qu'il éprouve pour le caractère mécanique de la fancy, dénigrée par Coleridge en faveur de l'organicité de l'imagination. Je me contenterai ici d'avancer que, de mon point de vue, l'aspect fragmentaire de la fancy peut être lu comme la signature esthétique d'un Hawthorne incurablement sceptique à l'égard de la transcendance revendiquée par un certain romantisme. The Marble Faun est par ailleurs exemplaire (a case in point) de cette attirance pour la ruine et la fragmentation. Il n'y a pas de consensus sur cette question dans la critique hawthornienne, mais je rapprocherais volontiers cette affection "contre-nature " pour la fancy du penchant allégorique dont sont marquées la plupart des fictions hawthorniennes (voir notamment « Rappaccini's Daughter », dont l'action se déroule également en Italie).

\section{RÉSUMÉS}

The Marble Faun s'achève sur le refus poli d'un personnage qui renâcle à satisfaire la curiosité du narrateur. Quand celui-ci lui demande si les oreilles du jeune Donatello sont aussi pointues que celles du Faune de Praxitèle, Kenyon sourit mystérieusement et répond : « On that point, at all events, there shall be no word of explanation. " Sur ce point, ou plutôt sur la pointe des oreilles de Donatello, l'« auteur " reste muet dans l'espoir, écrit-il, de situer cette créature aberrante «entre le réel et le fantastique » sans avoir à spécifier la place qu'elle occupe au sein de la taxonomie établie par Cuvier. La divulgation d'un petit détail anatomique menace de faire imploser l'édifice de la fiction hawthornienne. Le point en question, cependant, n'est pas simplement l'extrémité à partir de laquelle l'animal s'avère mesurable mais également la limite insaisissable (le punctum) qui met en péril la logique de la classification cuviérienne. En se penchant sur la métrique approximative de The Marble Faun, cet article se propose d'étudier la manière dont la romance imagine le point de fuite de la relation entre l'humain et l'animal.

Nathaniel Hawthorne's The Marble Faun ends with one character's polite refusal to satisfy the narrator's curiosity concerning young Donatello's possible animality. When asked if Donatello's 
ears are as pointy as those of the book's titular figure, the Faun of Praxiteles, Kenyon smiles inscrutably and responds: "On that point, at all events, there shall be no word of explanation." On that "point" - that of Donatello's ears-the author remains obstinately tight-lipped, having "hoped to mystify this anomalous creature between the Real and the Fantastic [...] without impelling him to ask how Cuvier would have classified poor Donatello." The disclosure of a small anatomical detail threatens to shatter the whole edifice of Hawthorne's fiction. The point in question here, however, is not just the extremity that makes measurement possible but also the ungraspable limit (the punctum) that undoes the logic of Cuvierian classification. Analyzing the metrics of The Marble Faun, my paper will query how the romance envisions the vanishing point of the human/animal relation.

INDEX

Keywords : Cuvier, epistemophilia, epistemophobia, point of view, taxonomy, The Marble Faun Mots-clés : Cuvier, épistémophilie, épistémophobie, point de vue, taxonomie, The Marble Faun

\section{AUTEUR}

\section{ANTOINE TRAISNEL}

Brown University 